\title{
Accuracy Limitations of the Locally One-Dimensional FDTD Technique
}

\author{
Ana Grande, José A. Pereda, Member, IEEE
}

\begin{abstract}
While the alternating-direction implicit finitedifference time-domain (ADI-FDTD) method preserves the second-order temporal accuracy of the conventional FDTD technique, the locally one-dimensional (LOD)-FDTD method exhibits a first-order in time splitting error. Despite this difference, the numerical dispersion analyses of these methods reveal that both present similar accuracy properties. For this reason, the characteristic non-commutativity error of the LOD-FDTD scheme has not received much attention. In this work, we determine the closed form of the local truncation error for the 3D-LOD-FDTD scheme. We find that it presents error terms which depend on the time-step size multiplied by the spatial derivatives of the fields. Numerical results confirm that these terms become a significant source of error which is not revealed in the dispersion analyses.
\end{abstract}

Index Terms-Alternating direction implicit split-step FDTD methods, local truncation error, locally one-dimensional FDTD method, numerical dispersion.

\section{INTRODUCTION}

The finite-difference time-domain (FDTD) method is a widely used tool in computational electrodynamics. However, it is a conditionally stable technique since the maximum timestep size is limited by the Courant condition. For this reason, in problems where the size of the geometrical features is much smaller than the wavelength, the Courant condition enforces small time-step sizes that over-sample the signal, making the FDTD method computationally inefficient.

Implicit techniques, such as the Crank Nicolson (CN)FDTD scheme, remove the Courant constraint [1]. Nevertheless, although the $\mathrm{CN}$ scheme presents a high degree of accuracy, it requires an extremely large sparse matrix to be solved, making the $\mathrm{CN}$ approach barely usable for practical problems. By contrast, Split-Step (SS)-FDTD techniques, such as the alternating-direction implicit (ADI)- and the locally onedimensional (LOD)-FDTD methods [2]-[6], exhibit tridiagonal linear systems meeting the requirements of unconditional stability at an acceptable computational cost.

The ADI-FDTD scheme preserves the second-order temporal accuracy. Indeed, it can be viewed as an $\mathcal{O}\left(\Delta_{t}^{2}\right)$ perturbation of the CN-FDTD method [7]. The LOD-FDTD scheme is more efficient than the ADI-FDTD due to its simpler updating procedures, although it presents an $\mathcal{O}\left(\Delta_{t}\right)$ splitting error [1].

Although the two approaches present different accuracies in time, both lead to the same numerical dispersion relation

Ana Grande is with the Dpto. de Electricidad y Electrónica, Universidad de Valladolid, 47011 Valladolid, Spain (email: agrande@uva.es). José A. Pereda is with the Dpto. de Ingeniería de Comunicaciones, Universidad de Cantabria, 39005 Santander, Spain.

Manuscript received XXXXX. This work was supported by the Spanish Ministerio de Economía y Competitividad under projects TEC2010-21496C03-01 and CONSOLIDER CSD2008-00066 and the subprogram Ramón y Cajal RYC-2010-06922.
[6], [9], [10]. As a result, in the framework of plane waves, both schemes yield similar numerical results. For this reason, the LOD-FDTD scheme is frequently claimed to provide the same accuracy with less computational expenditure than the ADI-FDTD technique [5], [11].

In this work, we examine in detail in what scenario the firstorder splitting error of the LOD-FDTD scheme plays a critical role and leads to a significant loss in accuracy. To this end, in section II, we review the formulation and the accuracy of the $\mathrm{CN}$ - and the ADI-FDTD methods [7]. We then calculate the local truncation error of the 3D-LOD-FDTD scheme and compare it to the corresponding errors of the $\mathrm{CN}$ - and the ADI-FDTD approaches. We find that the LOD-FDTD method exhibits extra error terms that are not present in ADI- or CNFDTD. These problematic terms are proportional to the timestep size and to the magnitude of spatial derivatives. Moreover, we show how for the two dimensional (2D) case the LODFDTD scheme can be expressed as an $\mathcal{O}\left(\Delta_{t}\right)$ perturbation of the CN-FDTD method. Finally, in section III, we illustrate the influence of the aforementioned errors, simulating a WR75 waveguide cavity.

\section{Formulation AND ACCURACY ANALYsis}

Three dimensional (3D) Maxwell curl equations in lossless isotropic media can be expressed as

$$
\frac{\partial \overrightarrow{\mathcal{U}}}{\partial t}-[\mathcal{R}] \overrightarrow{\mathcal{U}}=0
$$

where $\overrightarrow{\mathcal{U}}$ is the composite field vector given by

$$
\overrightarrow{\mathcal{U}}=\left(\mathcal{E}_{x}, \mathcal{E}_{y}, \mathcal{E}_{z}, \mathcal{H}_{x}, \mathcal{H}_{y}, \mathcal{H}_{z}\right)^{T}
$$

and the superscript $T$ denotes matrix transpose. The matrix $[\mathcal{R}]$ can be written as the sum of two operators

$$
[\mathcal{R}]=[\mathcal{A}]+[\mathcal{B}]
$$

where $[\mathcal{A}]$ and $[\mathcal{B}]$ are $6 \times 6$ block matrices defined as

$$
[\mathcal{A}]=\left(\begin{array}{cc}
0 & \frac{1}{\epsilon}\left[\mathcal{R}_{\mathrm{e}}\right] \\
-\frac{1}{\mu}\left[\mathcal{R}_{\mathrm{o}}\right] & 0
\end{array}\right),[\mathcal{B}]=\left(\begin{array}{cc}
0 & \frac{1}{\epsilon}\left[\mathcal{R}_{\mathrm{o}}\right] \\
-\frac{1}{\mu}\left[\mathcal{R}_{\mathrm{e}}\right] & 0
\end{array}\right)
$$

with $\left[\mathcal{R}_{\mathrm{e}}\right]$ and $\left[\mathcal{R}_{\mathrm{o}}\right]$ given by

$$
\left[\mathcal{R}_{\mathrm{e}}\right]=\left(\begin{array}{ccc}
0 & 0 & \frac{\partial}{\partial y} \\
\frac{\partial}{\partial z} & 0 & 0 \\
0 & \frac{\partial}{\partial x} & 0
\end{array}\right),\left[\mathcal{R}_{\mathrm{o}}\right]=\left(\begin{array}{ccc}
0 & -\frac{\partial}{\partial z} & 0 \\
0 & 0 & -\frac{\partial}{\partial x} \\
-\frac{\partial}{\partial y} & 0 & 0
\end{array}\right)
$$




\section{A. CN-FDTD Scheme}

Using the $\mathrm{CN}$ formalism, (1) can be approximated by

$$
\frac{\vec{U}^{n+1}-\vec{U}^{n}}{\Delta_{t}}-\mathbf{R} \frac{\vec{U}^{n+1}+\vec{U}^{n}}{2}=0
$$

where $\mathbf{R}$ represents the finite-difference version of $[\mathcal{R}]$ and $\vec{U}^{n}$ is the numerical field vector that approximates the exact field vector $\overrightarrow{\mathcal{U}}\left(i \Delta_{x}, j \Delta_{y}, k \Delta_{z}, n \Delta_{t}\right)$.

The truncation error of the CN-FDTD scheme can be derived substituting the exact solution $\overrightarrow{\mathcal{U}}$ of (1) into (4). Then, expanding in Taylor series and considering only the secondorder terms of the truncation error we obtain

$$
\overrightarrow{\mathcal{T}}_{C N}=\mathbf{E}_{\mathrm{CN}} \overrightarrow{\mathcal{U}}\left(\vec{r}_{m}, t_{m}\right)
$$

where the matrix $\mathbf{E}_{\mathrm{CN}}$ is given by

$$
\mathbf{E}_{\mathrm{CN}}=\left(\begin{array}{ccc|ccc}
2 h_{t} & & & & \frac{-h_{z}}{\epsilon} & \frac{h_{y}}{\epsilon} \\
& 2 h_{t} & & \frac{h_{z}}{\epsilon} & \frac{-h_{x}}{\epsilon} \\
& & 2 h_{t} & \frac{-h_{y}}{\epsilon} & \frac{h_{x}}{\epsilon} & \\
\hline \frac{-h_{z}}{\mu} & \frac{h_{z}}{\mu} & \frac{-h_{y}}{\mu} & 2 h_{t} & & \\
\frac{h_{y}}{\mu} & \frac{-h_{x}}{\mu} & & & 2 h_{t} & \\
& & & & 2 h_{t}
\end{array}\right)
$$

being

$$
h_{u}=-\frac{\Delta_{u}^{2}}{24} \frac{\partial^{3}}{\partial u^{3}}
$$

and

$$
\begin{aligned}
\overrightarrow{\mathcal{U}}\left(\vec{r}_{m}, t_{m}\right)= & \left(\mathcal{E}_{x}\left(\vec{r}_{1}, t_{1}\right), \mathcal{E}_{y}\left(\vec{r}_{2}, t_{2}\right), \mathcal{E}_{z}\left(\vec{r}_{3}, t_{3}\right),\right. \\
& \left.\mathcal{H}_{x}\left(\vec{r}_{4}, t_{4}\right), \mathcal{H}_{y}\left(\vec{r}_{5}, t_{5}\right), \mathcal{H}_{z}\left(\vec{r}_{6}, t_{6}\right)\right)^{T}
\end{aligned}
$$

with $t_{m} \in\left(n \Delta_{t},(n+1) \Delta_{t}\right)$ and with $\vec{r}_{m}$ inside an ellipse of center $\left(i \Delta_{x}, j \Delta_{y}, k \Delta_{z}\right)$ and semi-axes $\left(\Delta_{x} / 2, \Delta_{y} / 2, \Delta_{z} / 2\right)$.

Hence, in view of (6), the truncation error $\overrightarrow{\mathcal{T}}_{C N}$ depends on the square of the time-step and the spatial-cell sizes multiplied by the derivatives of the fields respect to the time and the spatial components, respectively.

\section{B. ADI-FDTD Scheme}

The ADI-FDTD approach is obtained by splitting (1) into two substeps. The two updating procedures in the main iteration are

$$
\begin{aligned}
\left(\mathbf{I}-\frac{\Delta_{t}}{2} \mathbf{A}\right) \vec{U}^{n+\frac{1}{2}} & =\left(\mathbf{I}+\frac{\Delta_{t}}{2} \mathbf{B}\right) \vec{U}^{n} \\
\left(\mathbf{I}-\frac{\Delta_{t}}{2} \mathbf{B}\right) \vec{U}^{n+1} & =\left(\mathbf{I}+\frac{\Delta_{t}}{2} \mathbf{A}\right) \vec{U}^{n+\frac{1}{2}}
\end{aligned}
$$

where $\mathbf{A}$ and $\mathbf{B}$ represent the finite-difference version of $[\mathcal{A}]$ and $[\mathcal{B}]$ with $\mathbf{R}=\mathbf{A}+\mathbf{B}$, and where $\mathbf{I}$ is the $6 \times 6$ identity matrix.

Eliminating the intermediate variable $\vec{U}^{n+\frac{1}{2}}$ in equation (8) we obtain

$$
\left(I+\frac{\Delta_{t}^{2}}{4} \mathbf{A B}\right) \frac{\vec{U}^{n+1}-\vec{U}^{n}}{\Delta_{t}}-\mathbf{R} \frac{\vec{U}^{n+1}+\vec{U}^{n}}{2}=0 .
$$

Comparing (9) and (4), we see that the ADI-FDTD formulation can be interpreted as an $\mathcal{O}\left(\Delta_{t}^{2}\right)$ perturbation of the $\mathrm{CN}$ FDTD scheme [7].
Following the procedure described in [7], the local truncation error of the ADI-FDTD scheme can be written as

$$
\overrightarrow{\mathcal{T}}_{A D I}=\left(\mathbf{E}_{\mathrm{CN}}+\mathbf{E}_{\mathrm{ADI}}\right) \overrightarrow{\mathcal{U}}\left(\vec{r}_{m}, t_{m}\right)
$$

where $\overrightarrow{\mathcal{U}}\left(\vec{r}_{m}, t_{m}\right)$ was given in (7) and $\mathbf{E}_{\mathrm{CN}}$ was presented in (5). The matrix $\mathbf{E}_{\mathrm{ADI}}$ contains the error terms unique to the ADI-FDTD algorithm. It can be written as

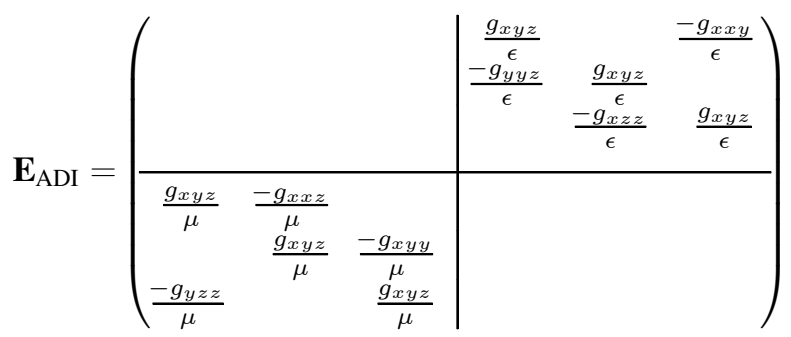

being

$$
g_{u v w}=-\frac{\Delta_{t}^{2}}{4 \epsilon \mu} \frac{\partial^{3}}{\partial u \partial v \partial w} .
$$

These error terms differ from those given in (6), since they depend on the product of the factor $\Delta_{t}^{2}$ and the spatial derivatives of the fields.

\section{LOD-FDTD Scheme}

1) Three dimensional case: The LOD-FDTD equations are obtained by splitting (1) into two substeps as follows

$$
\begin{aligned}
\left(\mathbf{I}-\frac{\Delta_{t}}{2} \mathbf{A}\right) \vec{U}^{n+\frac{1}{2}} & =\left(\mathbf{I}+\frac{\Delta_{t}}{2} \mathbf{A}\right) \vec{U}^{n} \\
\left(\mathbf{I}-\frac{\Delta_{t}}{2} \mathbf{B}\right) \vec{U}^{n+1} & =\left(\mathbf{I}+\frac{\Delta_{t}}{2} \mathbf{B}\right) \vec{U}^{n+\frac{1}{2}} .
\end{aligned}
$$

From (13) the LOD-FDTD scheme can be written as

$$
\begin{aligned}
& \frac{\vec{U}^{n+1}-\vec{U}^{n}}{\Delta_{t}}-\mathbf{R} \frac{\vec{U}^{n+1}+\vec{U}^{n}}{2} \\
& +\frac{\Delta_{t}}{4}\left(\mathbf{A B} \vec{U}^{n+1}-\mathbf{B} \mathbf{A} \vec{U}^{n}\right)+\frac{\Delta_{t}}{4}(\mathbf{A B}-\mathbf{B} \mathbf{A}) \vec{U}^{n+\frac{1}{2}}=0 .
\end{aligned}
$$

The local truncation error is then calculated as in the previous cases obtaining

$$
\overrightarrow{\mathcal{T}}_{L O D}=\left(\mathbf{E}_{\mathrm{CN}}+\mathbf{E}_{\mathrm{LOD}}\right) \overrightarrow{\mathcal{U}}\left(\vec{r}_{m}, t_{m}\right)
$$

where $\overrightarrow{\mathcal{U}}\left(\vec{r}_{m}, t_{m}\right)$ was given in (7) and $\mathbf{E}_{\mathrm{CN}}$ in (5). The matrix $\mathbf{E}_{\mathrm{LOD}}$ containing the error terms unique to the LOD-FDTD approach can be written as

$$
\mathbf{E}_{\mathrm{LOD}}=\left(\begin{array}{ccc|ccc} 
& f_{x y} & -f_{x z} & & \frac{g_{x x z}}{2 \epsilon} & \frac{-g_{x x y}}{2 \epsilon} \\
-f_{x y} & & f_{y z} & \frac{-g_{y y z}}{2 \epsilon} & & \frac{g_{x y y}}{2 \epsilon} \\
f_{x z} & -f_{y z} & & \frac{-g_{x z z}}{2 \epsilon} & \\
\hline & \frac{-g_{x x z}}{2 \mu} & \frac{g_{x x y}}{2 \mu} & & -f_{x y} & f_{x z} \\
\frac{g_{y y z}}{2 \mu} & & \frac{-g_{x y y}}{2 \mu} & f_{x y} & & -f_{y z} \\
\frac{-g_{y z z}}{2 \mu} & \frac{g_{x z z}}{2 \mu} & & -f_{x z} & f_{y z} &
\end{array}\right)
$$

where $g_{u v w}$ was given in (12) and $f_{u v}$ is defined as

$$
f_{u v}=-\frac{\Delta_{t}}{2 \epsilon \mu} \frac{\partial^{2}}{\partial u \partial v} .
$$


Thus, the LOD-FDTD scheme introduces additional first-order error terms that depend on the time-step size and the spatial derivatives of the fields. It will be shown in the results section that these error terms will be dominant in those regions with strong spatial variation of the fields, e. g. around singularities associated with corners or near-field sources.

2) Two dimensional case: For the $\mathrm{TE}_{z}$ polarization $\overrightarrow{\mathcal{U}}=\left(\mathcal{E}_{x}, \mathcal{E}_{y}, \mathcal{H}_{z}\right)^{T}$, and for the $\mathrm{TM}_{z}$ case $\overrightarrow{\mathcal{U}}=\left(\mathcal{E}_{z}, \mathcal{H}_{x}, \mathcal{H}_{y}\right)^{T}$. In both cases, the matrix $[\mathcal{R}]$ is reduced to a $3 \times 3$ matrix that can be decomposed as the sum of two operators, $[\mathcal{A}]$ and $[\mathcal{B}]$. These operators can be obtained particularizing (3) for the $\mathrm{TE}_{z}$ and the $\mathrm{TM}_{z}$ polarizations.

It can be seen that, for the $\mathrm{TE}_{z}$ and the $\mathrm{TM}_{z}$ cases, the following equality holds

$$
(\mathbf{A B}-\mathbf{B A}) \vec{U}^{n+\frac{1}{2}}=\mathbf{A B} \vec{U}^{n}-\mathbf{B} \mathbf{A} \vec{U}^{n+1}
$$

where $\mathbf{A}, \mathbf{B}$ and $\vec{U}$ are the numerical counterpart of $[\mathcal{A}],[\mathcal{B}]$ and $\overrightarrow{\mathcal{U}}$ for each polarization. Substituting (17) into (14) we thus obtain

$$
\frac{\vec{U}^{n+1}-\vec{U}^{n}}{\Delta_{t}}-\left[\mathbf{R}-\frac{\Delta_{t}}{2}(\mathbf{A B}-\mathbf{B A})\right] \frac{\vec{U}^{n+1}+\vec{U}^{n}}{2}=0 .
$$

Comparing this expression to (4), it is straightforward to see that the 2D-LOD-FDTD scheme can be recognized as an $\mathcal{O}\left(\Delta_{t}\right)$ perturbation of the CN-FDTD approach.

a) $T E_{z}-L O D-F D T D$ : Substituting the exact field solution $\overrightarrow{\mathcal{U}}$ of (1) into (18) and using the Taylor series we obtain the truncation error of the $\mathrm{TE}_{z}$-LOD-FDTD scheme

$$
\overrightarrow{\mathcal{T}}_{\text {LOD-TE }}=\left(\mathbf{E}_{\mathrm{CN}_{z}-\mathrm{TE}_{z}}+\mathbf{E}_{\mathrm{LOD}_{-\mathrm{TE}}}\right) \overrightarrow{\mathcal{U}}\left(\vec{r}_{m}, t_{m}\right)
$$

where $\mathbf{E}_{\mathrm{CN}-\mathrm{TE}_{z}}$ can be obtained particularizing (5) for the $\mathrm{TE}_{z}$ case and $\mathbf{E}_{\mathrm{LOD}-\mathrm{TE}_{z}}$ is the matrix that contains the error terms unique to the $\mathrm{TE}_{z}$-LOD-FDTD scheme. Thus, the local truncation error for the $\mathrm{TE}_{z}$ case can be written as

$$
\overrightarrow{\mathcal{T}}_{\text {LOD-TE }}=\left(\begin{array}{ccc}
2 h_{t} & f_{x y} & \frac{h_{y}}{\epsilon} \\
-f_{x y} & 2 h_{t} & -\frac{h_{x}}{\epsilon} \\
\frac{h_{y}}{\mu} & -\frac{h_{x}}{\mu} & 2 h_{t}
\end{array}\right) \overrightarrow{\mathcal{U}}\left(\vec{r}_{m}, t_{m}\right)
$$

where $h_{u}$ was given in equation (6) and $\overrightarrow{\mathcal{U}}\left(\vec{r}_{m}, t_{m}\right)=\left(\mathcal{E}_{x}\left(\vec{r}_{1}, t_{1}\right), \mathcal{E}_{y}\left(\vec{r}_{2}, t_{2}\right), \mathcal{H}_{z}\left(\vec{r}_{3}, t_{3}\right)\right)^{T} \quad$ with $t_{m} \in\left(n \Delta_{t},(n+1) \Delta_{t}\right)$ and $\vec{r}_{m}$ is inside an ellipse of center $\left(i \Delta_{x}, j \Delta_{y}\right)$ and semi-axes $\left(\Delta_{x} / 2, \Delta_{y} / 2\right)$.

In (20), the boxed terms correspond to the $\mathbf{E}_{\mathrm{LOD}-\mathrm{TE}_{z}}$ matrix. When those terms are set to zero, the matrix in (20) is reduced to $\mathbf{E}_{\mathrm{CN}-\mathrm{TE}_{z}}$. It should be noted that, unlike $\mathbf{E}_{\mathrm{LOD}}$, the matrix $\mathbf{E}_{\mathrm{LOD}_{-T E}}$ only contains first-order error terms in time. This result is in agreement with (18).

b) $T M_{z}-L O D-F D T D$ : For the $\mathrm{TM}_{z}$ polarization the local truncation error can be expressed as

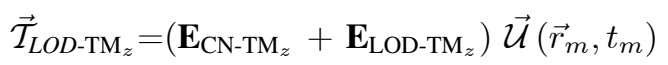

where $\mathbf{E}_{\mathrm{CN}-\mathrm{TM}_{z}}$ can be obtained by particularizing (5) for the $\mathrm{TM}_{z}$ case and $\mathbf{E}_{\mathrm{LOD}-\mathrm{TM}}$ contains the error terms unique to the $\mathrm{TM}_{z}$-LOD-FDTD scheme. The error for the $\mathrm{TM}_{z}$ case

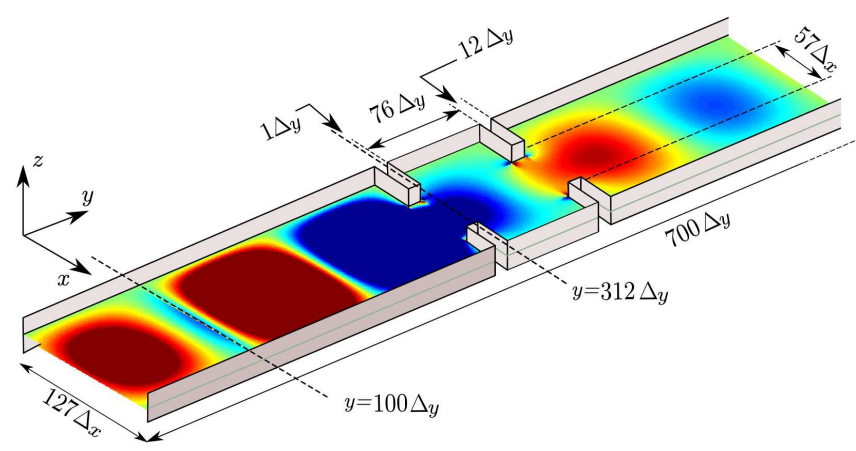

Fig. 1. Geometry of the problem simulated. Snapshot of the electric field computed by means of the LOD-FDTD technique in part of the simulation domain $\left(N_{\lambda}=205.77, N_{t}=45.2\right)$.

can thus be written as [13]

$$
\overrightarrow{\mathcal{T}}_{\text {LOD-TM }}=\left(\begin{array}{ccc}
2 h_{t} & -\frac{h_{y}}{\epsilon} & \frac{h_{x}}{\epsilon} \\
-\frac{h_{y}}{\mu} & 2 h_{t} & -f_{x y} \\
\frac{h_{x}}{\mu} & f_{x y} & 2 h_{t}
\end{array}\right) \overrightarrow{\mathcal{U}}\left(\vec{r}_{m}, t_{m}\right)
$$

where $h_{u}$ was given in equation (6) and $\overrightarrow{\mathcal{U}}\left(\vec{r}_{m}, t_{m}\right)=\left(\mathcal{E}_{z}\left(\vec{r}_{1}, t_{1}\right), \mathcal{H}_{x}\left(\vec{r}_{2}, t_{2}\right), \mathcal{H}_{y}\left(\vec{r}_{3}, t_{3}\right)\right)^{T} \quad$ with $t_{m} \in\left(n \Delta_{t},(n+1) \Delta_{t}\right)$ and $\vec{r}_{m}$ is inside an ellipse of center $\left(i \Delta_{x}, j \Delta_{y}\right)$ and semi-axes $\left(\Delta_{x} / 2, \Delta_{y} / 2\right)$.

The boxed terms correspond to the $\mathbf{E}_{\mathrm{LOD}-\mathrm{TM}_{z}}$ matrix. Again, unlike $\mathbf{E}_{\mathrm{LOD}}$, the matrix $\mathbf{E}_{\mathrm{LOD}-\mathrm{TM}_{z}}$ only contains first-order error terms in time, since the 2D-LOD-FDTD algorithm can be seen as a first-order perturbation of the CN-FDTD.

\section{NUMERICAL RESULTS}

To illustrate the influence of the error terms calculated in the previous section we have considered a WR75 waveguide cavity. The geometry of the problem is shown in Fig. 1. To simulate the propagation of the $\mathrm{TE}_{10}$ mode we have introduced a modal source located at $y=100 \Delta_{y}$. The source was a modulated Gaussian pulse with central frequency $f_{0}=12.5$ $\mathrm{GHz}$ and effective lateral bandwidth $\mathrm{BW}=2.5 \mathrm{GHz}$. The cell size was $\Delta_{x}=\Delta_{y}=\Delta=0.15 \mathrm{~mm}$ and the spatial resolution was $N_{\lambda}=\lambda_{g} / \Delta=205.77$, with $\lambda_{g}$ being the wavelength in the waveguide at the central frequency $f_{0}$. The ports of the waveguide were terminated by second-order one-way waveequation $\mathrm{ABCs}$ based on the Higdon operator [12].

We have simulated the propagation of the fundamental mode and computed the resonant frequency. To estimate the error, we have considered the solution obtained with the conventional FDTD method as a benchmark. In this case, the cell size was $\Delta / 4=0.0375 \mathrm{~mm}$ and the time step was the maximum allowed by the stability condition $\Delta_{t_{0}}=0.08845 \mathrm{ps}$. The resonant frequency of the dominant mode computed by means of the FDTD simulation was $f=12.789 \mathrm{GHz}$. Fig. 2 shows the absolute error of the results obtained with ADI and LOD as a function of the temporal resolution $N_{t}=T_{0} / \Delta_{t}$, being $T_{0}$ the wave period at the frequency $f_{0}$. We can see how both approaches provide practically the same solution. The slope of the curves confirms second-order convergence in time of the solution. The overall accuracy reached is limited by the value of the spatial resolution $N_{\lambda}$ that controls the spatial error. 


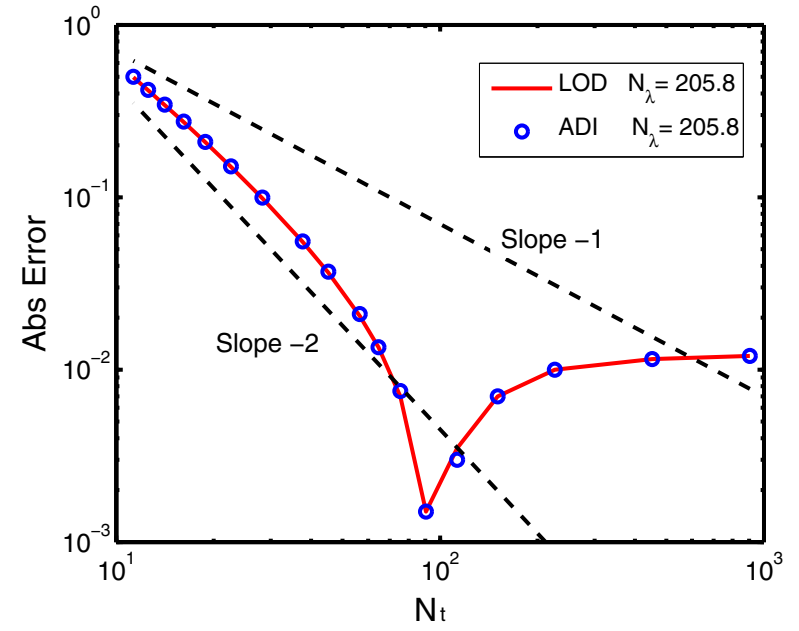

Fig. 2. Absolute error of the resonant frequency as a function of $N_{t}$. ADI and LOD provide the same solution. Convergence follows a second-order rate in time. The spatial resolution $N_{\lambda}=205.77$ limits the accuracy achieved.

Although not shown explicitly here, the ADI and the LOD techniques yield the same scattering parameters in the whole frequency band, from $10 \mathrm{GHz}$ to $15 \mathrm{GHz}$. This result is linked to the fact that both schemes present the same numerical dispersion relation [6], [9]. However, as indicated by the error analysis presented in section II, the ADI-FDTD scheme is second-order accurate in time while the LOD-FDTD method is only first-order accurate. Thus, in order to show the influence of the first-order error terms obtained in (22), we have computed the field pattern of the $E_{z}$ component at the plane $y=312 \Delta_{y}$ (see Fig. 1). The field pattern calculated by using the LOD-FDTD, the ADI-FDTD and the conventional FDTD schemes are shown in Fig. 3. It can be seen that, near the iris edges, where the fields present a strong spatial variation, the LOD-FDTD scheme leads to major errors. However, as we move away from the irises, and for a given value of $N_{\lambda}$ and $N_{t}$, the field values given by the ADI- and the LOD-FDTD coincide. The degradation of the LOD-FDTD solution near the iris corners can also be seen in the snapshot shown in Fig. 1.

\section{CONCLUSION}

The LOD-FDTD method achieves better CPU-time efficiency than the ADI-FDTD. In addition, numerical dispersion analyses reveal that it provides similar results to the ADIFDTD. Nevertheless, the LOD-FDTD scheme presents an $\mathcal{O}\left(\Delta_{t}\right)$ splitting error. In this work, the closed form of the local truncation error of the 3D-LOD-FDTD scheme is given. We find that it exhibits first-order in time error terms that depend on the time-step size multiplied by the spatial derivatives of the fields. These terms, which are not present in the ADI- or the CN-FDTD schemes, become a significant error source that does not show up in dispersion analysis. Moreover, for the two dimensional case, we find that the LOD-FDTD method can be expressed as an $\mathcal{O}\left(\Delta_{t}\right)$ perturbation of the CN-FDTD scheme.

Finally, we have simulated the propagation of the fundamental mode in a WR75 waveguide cavity. The results confirm that these error terms are critical when the fields

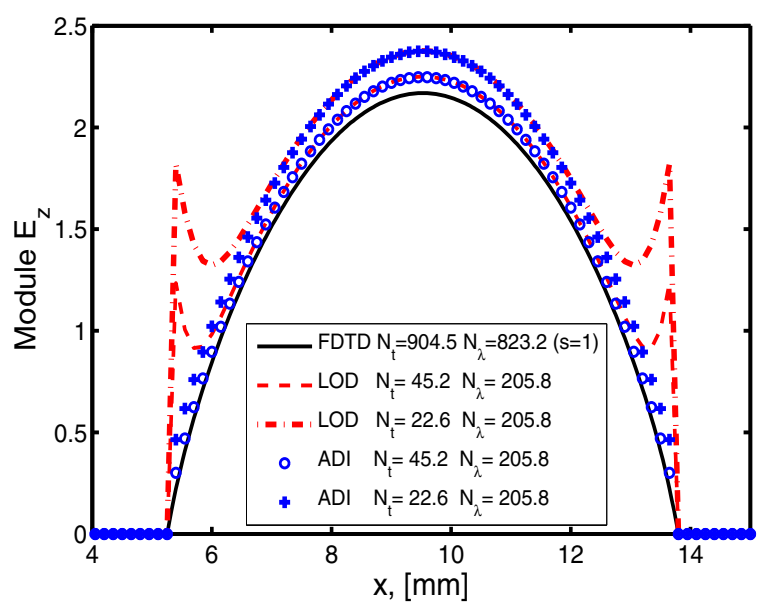

Fig. 3. Field pattern of the $E_{z}$ component at the plane $y=312 \Delta_{y}$ calculated by the LOD-FDTD, the ADI-FDTD and the conventional FDTD schemes.

present strong spatial variations. Hence, statements such as "The computational time is reduced with the accuracy being maintained, when compared with the ADI-FDTD..." made in [5] when talking about the LOD-FDTD method should be framed in an appropriate context.

\section{REFERENCES}

[1] G. I. Marchuk, Methods of Numerical Mathematics. New York: SpringerVerlang, 1975.

[2] T. Namiki, "A new FDTD algorithm based on alternating-direction implicit method," IEEE Trans. Microw. Theory Tech., vol. 47, no. 10, pp. 2003-2007, Oct. 1999.

[3] F. Zheng, Z. Chen, and J. Zhang, "Toward the development of a three-dimensional unconditionally stable finite-difference time-domain method," IEEE Trans. Microw. Theory Tech., vol. 48, no. 9, pp. 15501558, Sep. 2000.

[4] J. Lee and B. Fornberg, "A split step approach for the 3-D Maxwell's equations," J. Comput. Appl. Math., vol. 158, pp. 485-505, 2003.

[5] J. Shibayama, M. Muraki, J. Yamauchi and H. Nakano, "Efficient implicit FDTD algorithm based on locally one-dimensional scheme," Electron. Lett., vol. 41, no. 19, pp. 1046-1047, Sep. 2005.

[6] Q.-F. Liu, Z. Chen and W.-Y. Yin, "An arbitrary-order LOD-FDTD method and its stability and numerical dispersion," IEEE Trans. Antennas Propag., vol. 57, no. 8, pp. 2409-2417, Aug. 2009.

[7] S. G. Garcia, T.-W. Lee and S. C. Hagness, "On the accuracy of the ADI-FDTD method," IEEE Antennas Wireless Propag. Lett., vol. 1, pp. 31-34, 2002.

[8] E. L. Tan, "Fundamental schemes for efficient unconditionally stable implicit finite-difference time-domain methods," IEEE Trans. Antennas Propag., vol. 56, no. 1, pp. 170-177, Jan. 2008.

[9] F. Zheng and Z. Chen, "Numerical dispersion analysis of the unconditionally stable 3-D ADI-FDTD method," IEEE Trans. Microw. Theory Techn., vol. 49, pp. 1006-1009, May 2001.

[10] S. G. Garcia, R. G. Rubio, A. R. Bretones and R. G. Martín, "On the dispersion relation of ADI-FDTD," IEEE Microw. Wireless Compon. Lett., vol. 16, no. 6, pp. 354-356, Jun. 2006.

[11] J. Shibayama, M. Muraki, R. Takahashi, J. Yamauchi and H. Nakano, "Performance evaluation of several implicit FDTD methods for optical waveguide analyses," J. Lightw. Technol., vol. 24, no. 6, pp. 2465-2472, 2006.

[12] J. A. Pereda, A. Serroukh, A. Grande and A. Vegas, "Implementation of absorbing boundary conditions based on the second-order one-way wave equation in the LOD- and the ADI-FDTD methods," IEEE Antennas Wireless Propag. Lett., vol. 11, pp. 981-983, 2012.

[13] A. Grande, J. A. Pereda, A. Serroukh, I. Barba, A. C. L. Cabeceira and J. Represa, "Reinterpreting four-stage split-step FDTD methods as two-stage methods," IEEE Trans. Antennas Propag., vol. 61, no. 11, pp. 5818-5821, Nov. 2013. 